\title{
Goods Demand Forecast and Dispatching Plan in Aviation Emergency Rescue
}

\author{
Ming Zhang ${ }^{a}$, Hui Yu ${ }^{b}$, Shuo Wang $^{c}$, Xiangyu Li ${ }^{d}$, Ming Zhang ${ }^{\mathrm{e}}$, Yi Zhou ${ }^{\mathrm{f}}$ \\ College of Civil Aviation, Nanjing University of Aeronautics and Astronautics, Nanjing, 211106, \\ China
}

azhangming_nuaa@126.com, ${ }^{b}$ yuhui19920118@163.com, ${ }^{c} w 0913 s @ 163 . c o m,{ }^{d}$ linkinparklxy@qq.co m, 'zh_ming2014@163.com, ${ }^{\mathrm{f}}$ fczhouyi@163.com

Keywords: aviation emergency rescue, fuzzy clustering, Aircraft path planning, material distribution

\begin{abstract}
For the time-dependent nature of goods and materials demand and the complexity of dispatching plan in aviation rescue countering major natural disasters, different methods of buffering demands have been adopted to solve the problem of differences in demand for fast-moving consumer goods and durable goods and fuzzy clustering has been employed to divide the disaster-hit area and to determine the dispatching priority; at the end of this paper, problems in aircraft route planning and delivery scheme have been solved by establishing a planning model of goods dispatching and designing some corresponding solution algorithms, in this way a systemic reaction chain of aviation rescue has been built. Results of simulation analysis of examples have shown that this rescue chain is of remarkable effect in dealing with the dynamic forecast of goods and materials demand in disaster-hit area and the time-dependent nature in the whole rescue network and the complexity of large-scale coordinated dispatching.
\end{abstract}

\section{Introduction}

In recent years, major natural disasters such as the huge earthquake in Wenchuan, snow disaster in the south of China, the earthquake in Ya'an and the flood in Beichuan have exerted great threat for the personal and property safety of local people, therefore, sterner requirement has been raised for the rescue after disasters. Meanwhile, only the ground rescue cannot meet the requirement of complex rescue mission because of the complicated landform and severe environment. However, the participation of aviation rescue makes up of a 3-D rescue which has unique advantages such as rapidness, flexibility and being less limited by ground routes ${ }^{[1]}$.

Sophisticated aviation rescue systems have been built in the developed countries in Europe and America based on their own national conditions, so they have strong emergency rescue ability in the face of natural disasters and emergencies ${ }^{[2]}$.By contrast, being limited by low-altitude opening and navigation development issues, the aviation emergency rescue in China lags behind ${ }^{[3]}$. On problems of dispatching disaster-relief commodity, Barbarosoglu ${ }^{[4]}$, Chang ${ }^{[5]}$ and Beraldi ${ }^{[6]}$ have established corresponding planning models in the perspective of stochastic optimization; Araz ${ }^{[7]}$, Tzeng ${ }^{[8]}$ and Zhan Shalei ${ }^{[9]}$ have studied how to select sites for emergency vehicles and to dispatch goods and materials through multi-objective programming; Vanda ${ }^{[10]}$, Bodin ${ }^{[11]}$ and Yang Rongsheng ${ }^{[12]}$ studied from the aspect of making dispatching plan of aviation emergency rescue and decision making; Pssquier ${ }^{[13]}$, Zhangxin ${ }^{[14]}$, Xia Zhenghong ${ }^{[15]}$ and Liu Lang ${ }^{[16]}$ have proposed improvement for air-ground coordination and air line optimization by analyzing existing disadvantages of low efficiency in aviation rescue. However, these present studies have not put forward perfect scheme of dispatching goods and material and planning aircrafts.

In this paper, in the perspective of forecasting major goods - fast-moving consumer goods and durable goods dispatched by aircraft, the disaster-stricken areas are divided into different groups according to the situations of disaster through fuzzy clustering; and dispatching aircraft and delivery of relief supplies have been realized by setting up corresponding planning models; a systemic reaction chain of aviation rescue has been established by taking into all advantages into consideration. 


\section{Goods Demand Forcast}

In order to simplify the forecasting process and maintain the reasonability of forecast, following assumptions should be made at first: (1) number of disaster-hit areas and their positions and space distributions have been known (2) statistic data of situations in the areas can be obtained (3) time-varying goods demand is highly correlated with the number of survivals in the areas. Demand forecast formula is:

$$
D_{i}^{l}(t)=\left\{\begin{array}{l}
\max \left\{a^{l} \times \delta_{i}(t) \times L+z_{1-\alpha} \times S_{i}^{l}(t) \times \sqrt{L}, 0\right\}, l \in R_{\text {fmcg }} \\
\max \left\{a^{l} \times \delta_{i}(t)+b_{i}^{l}-\sum_{\varepsilon=1}^{t-1} A_{i}^{l}(t-\varepsilon), 0\right\}, l \in R_{\text {dura }}
\end{array}\right.
$$

In this formula, $D_{i}^{l}(t)$ refers to the quantity of time-dependent demand for goods $l$ in given time interval $t ; a^{l}$ and $b_{i}^{l}$ are the quantity of goods $l$ demanded by per person per hour and the corresponding buffering demand in disaster-hit area $i$ respectively and $l$ are fast-moving consumer goods and durable goods; $A_{i}^{l}(t-\varepsilon)$ is the quantity of time-dependent demand for goods $l$ in given time interval $t-\varepsilon$; $L$ is the presupposed upper limit value of goods dispatching for two times in succession in the area; $R_{\text {fmcg }}$ and $R_{\text {dura }}$ are the fast-moving consumer goods and durable goods; $z_{1-\alpha}$ is the selected statistic whose corresponding shortage rate of time-dependent goods demand is set as $\alpha ; \delta_{i}(t)$ is the estimated number of trapped people in the area $I$ at given time interval; $S_{i}^{l}(t)$ is the standard deviation of time-dependent demand quantity, which can be worked out in Formula (2):

$$
S_{i}^{l}(t)=\frac{\sqrt{\sum_{\varepsilon=0}^{t-1}\left[D_{i}^{l}(t-\varepsilon)-\overline{D_{i}^{l}(t)}\right]^{2}}}{t-1}
$$

In this formula, $\overline{D_{i}^{l}(t)}$ is the mean value of $D_{i}^{\prime}(t)$ at the time interval of $t$ and it is presented in the following formula:

$$
\overline{D_{i}^{l}(t)}=\frac{a^{l} \times \sum_{s=0}^{t-1} \delta_{i}^{l}(t-\varepsilon)}{t}
$$

From Formula (1), it can be seen that the main distinction of fast-moving consumer goods and durable goods demanded lies in the buffering demand resulting from supply failure because of potential goods shortage. For fast-moving consumer goods, the potential shortage of the two kinds of goods at $\mathrm{L}$ is $z_{1-\alpha} \times S_{i}^{l}(t) \times \sqrt{L}$; for any disaster-hit are $i$, the probability of goods shortage $\alpha$ and the maximum time interval of goods distribution need to meet the requirement of goods demand at $L$ (Formula (4)) while durable goods is not in continuous consumption, so a constant $b_{i}^{s}$ is set to deal with the problem of potential goods shortage.

$$
\operatorname{Prob}\left(Q \leq a^{l} \times \delta_{i}(t) \times L+z_{1-\alpha} \times S_{i}^{l}(t) \times \sqrt{L}\right)=1-\alpha
$$

\section{Disaster-hit Areas Division}

In order to make it convenient to allocate goods, multi-criteria fuzzy clustering is used to divide disaster-hit areas, which is of great importance for dynamic goods delivery with different supply-demand situations in each disaster-hit area: it is more effective to know how much goods needed in each area and to achieve better disaster relief. Detailed steps are as follows:

\subsection{Initial Data Processing}

By means of analyzing, 4 kinds of emergency properties below are used as the criteria of dividing.

1. $u_{i}^{1}(t)$ : is the difference value of current time from the last rescue time in disaster-hit area $i$. In the process of distribution, the larger difference means that it is more urgent for rescue.

2. $u_{i}^{2}(t)$ : is the proportion of casualty to the number of trapped people in time interval $t$ in $i$. In general, higher proportion means that it is more urgent for rescue. 
3. $u_{i}^{3}(t)$ : in time interval $t$ the proportion of the number of social vulnerable group to trapped people in $i$. Higher proportion means higher urgency for rescue.

4. $u_{i}^{4}(t)$ : the damage of building in $i$ in $t$. More buildings being damaged leads to more casualties. More serious damage means more urgent for rescue. Thus, a $4 \times 1$ discriminant vector of urgency property $_{U_{i}(t)}$ can be obtained:

$$
U_{i}(t)=\left[u_{i}^{1}(t), u_{i}^{2}(t), u_{i}^{3}(t), u_{i}^{4}(t)\right]^{\mathrm{T}}
$$

\subsection{Fuzzy Clustering Grouping}

(1) Binary system conversion: the main aim of this step is to change the vector $U_{i}(t)$ into binary data to facilitate the fuzzy clustering in next step. There are two another steps in this step:

Step 1: in any one disaster-hit area $i$, all urgency properties are expressed by 5 corresponding logical attributes which are "Very High, High, Medium, Low and Very Low ( VH, $H$, $M, L$ and $V L$ in short) based on the following rules of logical conversion (Table 1):

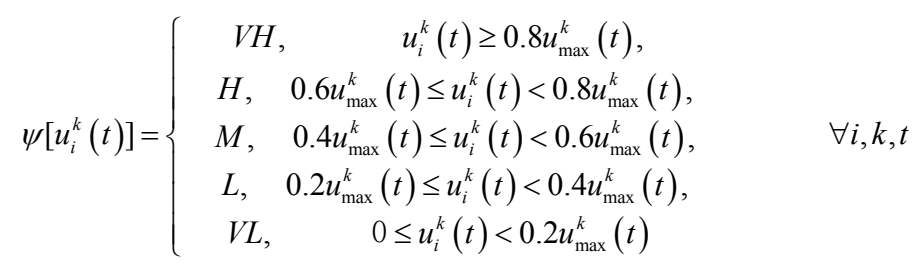

In this expression, $\psi\left[u_{i}^{k}(t)\right]$ is the logic measured value corresponding $u_{i}^{k}(t) ; u_{i}^{k}(t)$ is the initial data of $k$ urgency property in $t$ in the area $i ; u_{\max }^{k}(t)$ is the maximum expected value that can be got; $u_{i}^{1}(t), u_{i}^{2}(t)$ and $u_{i}^{3}(t)$ can be used in the formula above while $u_{i}^{4}(t)$ is not a attribute that can be quantized, so there is no need to converse it.

Step 2: the logical value is conversed as a binary value and the 4 logical indicators are expressed by 4 binary digit; that is to say, any urgency attribute can be changed into a 4-bit binary code $\psi_{j}^{k}(t)(j=1,2,3,4)$ according to conversion rules, (as shown in Formula (7)):

$$
\psi_{i}^{k}(t)=\left[\sigma_{i, 1}^{k}(t), \sigma_{i, 2}^{k}(t), \sigma_{i, 3}^{k}(t), \sigma_{i, 4}^{k}(t)\right]
$$

In addition, to facilitate data processing in practical application, any $\sigma_{i, j}^{k}(t)$ can be standardized and its corresponding standard value is $\overline{\sigma_{i, j}^{k}(t)}$ which is expressed as:

$$
\tilde{\sigma}_{j}^{k}(t)=\frac{\sigma_{i, j}^{k}(t)-\overline{\sigma_{j}^{k}(t)}}{\hat{\Lambda}^{k}\left(\sigma_{j}(t)\right]}
$$

In this formula, $\overline{\sigma_{j}^{k}(t)}$ and $S\left[\hat{\sigma}_{j}^{k}(t)\right]$ are the mean value and standard deviation of $\sigma_{i, j}^{k}(t)$ respectively. and the standard binary vector of urgency attribute is

$$
\tilde{\psi}_{i}^{k}(t)=\left[\tilde{\psi}_{i, 1}^{k}(t), \tilde{\psi}_{i, 2}^{k}(t), \tilde{\psi}_{i, 3}^{k}(t), \tilde{\psi}_{i, 4}^{k}(t)\right]
$$

Tab.1 Definitions of 4-bit binary codes for linguistic criteria

\begin{tabular}{ccccc}
\hline Logic index & $\sigma_{i, 1}^{k}(t)$ & $\sigma_{i, 2}^{k}(t)$ & $\sigma_{i, 3}^{k}(t)$ & $\sigma_{i, 4}^{k}(t)$ \\
\hline$V H$ & 1 & 1 & 1 & 1 \\
$H$ & 1 & 1 & 1 & 0 \\
$M$ & 1 & 1 & 0 & 0 \\
$L$ & 1 & 0 & 0 & 0 \\
$V L$ & 0 & 0 & 0 & 0 \\
\hline
\end{tabular}

(2) Establishing Fuzzy Matrix 
A $I \times I$ fuzzy incidence matrix $W(t)$ is produced according to the given number of disaster-hit area $I$ the assumption in the first step and the standard binary vector of urgency attribute. In this matrix, $w_{p q}(t)$ refers to how close disaster-hit area $p$ is to the disaster-hit area $q$, which can be expressed by a corresponding fuzzy number. Mathematical expressions of solving $W(t)$ and $w_{p q}(t)$ are:

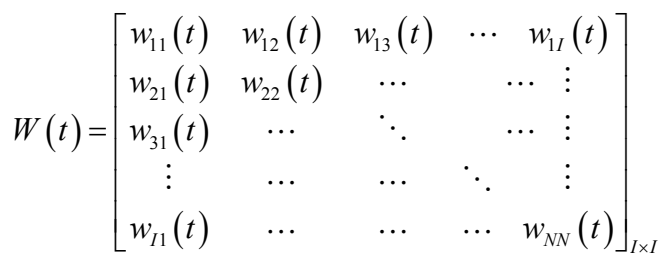

$$
\begin{aligned}
& w_{p q}(t)=1-\frac{1}{\beta} \sqrt{\sum_{k=1}^{4} \sum_{j=1}^{4}\left[\tilde{\sigma}_{p j}^{k}-\tilde{\sigma}_{q j}^{k}\right]^{2}} \forall t
\end{aligned}
$$

$\beta$ is the presupposed parameter values of $w_{p q}(t)$ ranging from 0 to 1 and $j$ is the given bit code. According to the ultimate principle of fuzzy clustering, the fuzzy incidence matrix $W(t)$ should be combined to meet the following requirement:

$$
\tilde{W}(t) \circ \tilde{W}(t)=\tilde{W}(t)
$$

$\widetilde{W}(t)$ is the form of combined fuzzy matrix of $W(t) . w_{p q}(t)$ in $W(t)$ should be minimax recombination - the pessimistic decision conversion, which is:

$$
w_{p q}=\max _{i=1}^{I}\left\{\min \left[w_{p i}(t), w_{i q}(t)\right]\right\}
$$

Repeat the iteration until Formula (12) meets the requirement in Formula (13).

(3) Clustering

The process of calculating, disaster-hit sites with similar degree of urgency are divided into one group to make the urgency attribute difference more obvious.

1.To initialize iteration steps. The starting time is set as the first time interval $(t=1)$; juxtapose row search index $\pi=1$; input combined fuzzy incidence matrix $\widetilde{W}(t)$; start the iteration from the first row (that is from $\tilde{w}_{1}(t)$ ) and make $p=\pi$. Make the most seriously stricken site as the one to be handled first and start the grouping. Any disaster-hit site that is first targeted can be regarded as the dividing criteria in their group.

2.For the given disaster-hit site (expressed as $p$ ), the row with $p$ in $\widetilde{W}(t)$ is removed (that is $\left.\tilde{w}_{p}(t)^{\mathrm{T}}\right)$. When a $\widetilde{w_{p}}(t)$ is targeted as the target customer, there is no need to re-cluster this site in corresponding time interval and $\tilde{w}_{p}(t)^{\mathrm{T}}$ should be removed to facilitate the following clustering.

3. To locate the maxim element in $\widetilde{w_{p}}(t)$ and mark it as $w_{p q}^{\max }(t)$ and then execute following steps in sequence: (1) If $_{w_{p q}^{\max }}(t)>\lambda_{1}$, put $q$ into the same group of $\mathrm{p}$ and remove $\tilde{w}_{p}(t)^{\mathrm{T}}$, which implies that $q$ and $p$ have the similar degree of urgency. That is why the two are put into one group and $\lambda_{1}$ is the cut-off value which can be set flexibly according to specific situation. (2) Return to Step 2 to further check elements in $\tilde{w}_{p}(t)$ until the other elements cannot meet the requirement of clustering above. If there are elements should be clustered, then to remove them. When all these elements are removed, the clustering of urgency attributes in $p$ in given time interval will be finished. (3) If $\mathrm{p}$ is selected as $q$ (that is $p=q$ ), go back to Step 1 to operate the clustering in corresponding sections in $\widetilde{W}(t)$ (4) Otherwise make $p=\pi$, and then do the clustering in the next disaster-hit area selected.

4.Termination rule: if the following requirements are met, all the algorithm will be terminated: (1) all the rows are conducted by clustering; (2) if not, return to Step 1 to do the next iteration.

For any given time interval $\mathrm{t}$, all disaster-hit areas will be divided into $G$ group after the steps 
above, when for the disaster-hit areas, a standard binary matrix of urgency attribute based on grouping can be got $-\tilde{\psi}_{g}(t)$ :

$$
\tilde{\psi}_{g}(t)=\left[\psi_{i g}(t), i_{g}=1,2, \cdots, l_{g}\right]_{16 \times l_{g}}
$$

$\tilde{\psi}_{g}(t)$ is a $\left(16 \times l_{g}\right)$ standard binary matrix of urgency attribute, which is composed by standard binary vectors of urgency attribute for all disaster-hit sites $i$ in Group $g$. And $l_{g}$ refers to the number of disaster-hit areas in the group. This $16 \times 1$ matrix can be expressed as:

$$
\psi_{i_{g}}(t)=\left[\widetilde{\sim}_{i_{g} j}^{k}(t), j=1, \cdots 4 ; k=1, \cdots 4\right]_{16 \times 1}
$$

\section{Models of Aircraft Route Planning and Goods Delivery}

\subsection{Model Description:}

With the further opening of low-altitude airspace and rapid development of general aviation in China, a national or local model of rescue has been established by using the aviation emergency rescue in developed countries for reference. In China, if disaster happens in a district, the aircraft will be dispatched to rescue for the divided area of responsibility. This model is based on scene, which means when the priority of disaster-hit area and quantity of goods needed in each dispatching cycle are determined, to maximize the satisfaction rate of goods supply in each disaster-stricken area and to minimize the fight of aircraft dispatched by scheduling the number and time of aircrafts dispatched.

\subsection{Model Assumptions}

(1) Relief goods are delivered to many disaster-hit areas in different groups from many distribution centers; the distribution centers and disaster-hit areas are known and the relief goods dispatched can meet the demand in disaster-hit areas.

(2) Relief goods needed can be loaded into one aircraft and the demand loading should within the maximum load of aircraft; the goods for each disaster-hit area should meet the minimum margin and be delivered by only one aircraft-delivery in batches are not allowed.

(3) The maximum load of each aircraft dispatched is fixed, so overload is not allowed; the delivery distance of each aircraft should be within the maximum distance; each aircraft should start from the distribution center and return to the center after delivering the relief goods.

(4) Aircrew dispatching and aircraft dispatching are dependent, so the former is not considered; aircrafts are refueled and loaded in the distribution center and unloaded in the disaster-hit area.

For any $g$, in given time interval $t$, to maximize the satisfaction rate in each disaster-hit area $-F_{g}^{1}(t)$ and to minimize the total delivery flight $-F_{g}^{2}(t)$. Their functions are:

$$
\begin{aligned}
& \max F_{g}^{1}(t)=\frac{\sum_{\forall l} \sum_{m=1}^{M} \sum_{\forall i g g g} X_{m, i g}^{l}(t)}{\sum_{\forall l}^{M} \sum_{m=1}^{M} \sum_{\forall i g \in g} D_{i g}^{l}(t)}, \forall(g, t) \\
& \min F_{g}^{2}(t)=\sum_{m=1}^{M}\left\{\sum_{k=1}^{K_{m}}\left[\sum_{i=1}^{n_{m k}} d_{r_{m i(i-1)} r_{m i l}}+d_{r_{m j m_{m k}} r_{m j 0}} \cdot \operatorname{sign}\left(n_{m k}\right)\right]\right\}
\end{aligned}
$$

$M$ is the number of distribution centers and $m$ is the index; $K_{m}$ is the number of aircrafts in $m$ and $k$ is the index; $i_{g}$ is the number of disaster-stricken areas in each group and $X_{m, i g}^{l}(t)$ is the decision variables referring to the quantity of goods $l$ delivered from $m$ to disaster-hit areas in $t$. $D_{i g}^{l}(t)$ is the quantity of goods demanded in the area and $n_{m k}$ is the number of disaster-hit area served by the $k$ aircraft in the distribution center $m$, and $i$ is the index; $R_{m j}$ is the $\mathrm{j}$ route within $\mathrm{m}$ and $r_{m j i}$ refers to the order of disaster-hit site $i_{g} \in g$ along $j$ in $m . \quad r_{m j 0}$ is seen as the delivery center, $d_{i_{g}, i_{g}}\left(i_{g}, i_{g}{ }^{\prime} \in g\right)$ the distance between disaster-hit areas and $d_{m, i_{g}}$ the distance from disaster-hit site 
to distribution center. The constraint condition is:

$$
\begin{aligned}
& \sum_{m=1}^{M} X_{m, i_{g}}^{l}(t) \leq D_{i_{g}}^{l}(t), \forall\left(g, i_{g}, l, t\right) \\
& \sum_{g=1}^{G} \sum_{\forall i_{g} \in g} \sum_{i=1, i, i i_{g}}^{n_{m i}} U_{l} \times X_{m, i}^{l} \leq Q_{m k}(\forall m, t) \\
& \quad d_{r_{m j}(i-1) r_{m i i}}+d_{r_{m j m_{m k}} r_{m j 0}} \cdot \operatorname{sign}\left(n_{m k}\right) \leq f_{m k} \cdot v_{m k} / c_{m k}\left(0 \leq n_{m k} \leq L_{m}\right) \forall m, k \\
& \sum_{k=1}^{K_{m}} n_{m k}=L_{m}(\forall m) \\
& \sum_{m=1}^{M} L_{m}=N \\
& R_{n j}=\left\{r_{n k i} \mid r_{n k i} \in\left\{1,2, \cdots, L_{m}\right\}, i=1,2, \cdots, n_{m k}\right\} \\
& R_{m k_{1}} \cap R_{m k_{2}}=\varnothing, \forall m k_{1} \neq m k_{2} \\
& \operatorname{sign}\left(n_{m k}\right)=\left\{\begin{array}{l}
1, n_{m k} \geq 1 \\
0, \mathrm{else}
\end{array}\right. \\
& X_{m, i_{g}}^{l}(t) \geq 0, \forall\left(g, i_{g}, l, m, t\right)
\end{aligned}
$$

$U_{l}$ is the unit volume of goods $l, Q_{m k}, f_{m k}, c_{m k}$ and $v_{m k}$ refer to the maximum load capacity, maximum fuel capacity, average fuel consumption rate and average flight speed respectively; $L_{m}$ refers to the number of disaster-hit sites rescued by distribution center $m$ and $N$ is the total number of disaster-hit areas.

Constraint condition (18) ensures that the total delivery to any disaster-hit site is within the real time-varying quantity of goods demanded; Constraint condition (19) ensures that no any aircraft is overloaded Constraint condition (20) ensures that the flight of any aircraft is within the normal maximum flight; (21) ensures that all the disaster-hit sites rescued by each distribution center are rescued (22) ensures that all disaster-hit areas are rescued (23) means the composition of disaster-hit site along each route; (24) asks that each disaster-hit area is rescued by only one aircraft; (25) refers to that when the number of disaster-hit area rescued by $k$ aircraft in $m$ is over 1, the aircraft participates the rescue work and $\operatorname{sign}\left(n_{m k}\right)=1$, otherwise, $\operatorname{sign}\left(n_{m k}\right)=0 ;(26)$ ensures that the goods delivered for any disaster-hit area at any time is of practical significance.

Analysis of Examples

Taking historical statistical data of serious disasters for reference, simulation data generated through random number generation are used in this paper.

\subsection{Calculation of Goods Demanded}

Calculation of quantity of fast-moving consumer goods and durable goods (Table 1 shows the parameter of goods demanded): in this paper, the former mainly includes drinking water and boxed meals and the latter mainly includes tents and sleeping bags (unit: $h$ ). parameter values are shown in Table 2:

Tab.2 Material needs-related parameters

\begin{tabular}{cccccccccc}
\hline \multicolumn{4}{c}{$a^{l}$} & \multicolumn{1}{c}{} & \multicolumn{1}{c}{$b_{i}^{l}$} & & $\alpha$ & $\bar{L} /(h)$ \\
\hline$a^{1}$ & $a^{2}$ & $a^{3}$ & $a^{4}$ & $b_{i}^{1}$ & $b_{i}^{2}$ & $b_{i}^{3}$ & $b_{i}^{4}$ & 0.05 & 6 \\
0.05 & 0.25 & 0.5 & 0.25 & 100 & 50 & 20 & 20 & & \\
\hline
\end{tabular}

\subsection{Disaster-hit Area Division}

According to processing data of casualty and statistical data in disaster-hit sites (select one time interval), based on the 4 urgency attributes, the division is displayed in Table 3: 
Tab.3 The emergency properties of affected points

\begin{tabular}{ccccc}
\hline $\begin{array}{c}\text { The number of } \\
\text { disaster-hit sites }\end{array}$ & $\begin{array}{c}\text { Population } \\
\text { size }\end{array}$ & $\begin{array}{c}\text { Number of } \\
\text { people in } \\
\text { stricken area }\end{array}$ & $\begin{array}{c}\text { The } \\
\text { number of } \\
\text { Casualties }\end{array}$ & $\begin{array}{c}\text { Proportion of brick } \\
\text { and Concrete } \\
\text { Structure }\end{array}$ \\
\hline 1 & 1572 & 230 & 127 & VH \\
2 & 2890 & 477 & 48 & $M$ \\
3 & 3874 & 961 & 159 & $V H$ \\
4 & 11149 & 3110 & 122 & $H$ \\
5 & 26581 & 8864 & 220 & $M$ \\
6 & 1746 & 293 & 71 & $H$ \\
7 & 1084 & 283 & 63 & $H$ \\
8 & 4385 & 1081 & 144 & $H$ \\
9 & 27759 & 8910 & 18 & $H$ \\
10 & 9330 & 2369 & 288 & LL \\
11 & 1764 & 327 & 26 & $V L$ \\
12 & 9881 & 2019 & 7 & 3 \\
13 & 3701 & 656 & 3 & \\
\hline
\end{tabular}

(1) Binary system conversion: change the statistical data in Formula (6) into logical data and produce the binary numerical table combining the table of bit code conversion;

(2) Fuzzy matrix generation: for an ideal grouping, that is to get a reasonable number of groups with obvious discrepancy, a suitable cut-off parameter $\lambda_{1}$ should be selected, which is 0.87 in this paper and the 13 disaster-hit sites should be divided into 3 or 4 groups; besides, as the criteria of grouping, the urgency attributes have no difference in importance degree and their weights are $\varpi_{1}, \varpi_{2}, \varpi_{3}$ and $\varpi_{4}$ which are 0.25 .

(3) Divide into groups: the goods demanded every 4 hours and the situation in each disaster-hit area are assessed and the grouping in the first day is presented. Experiment shows that the priority of each disaster-hit site remains unchanged, for example, the casualty and damage are relatively stable for some time after the disaster. In Table 4, the grouping in the first 3 rescue periods are displayed:

Tab.4 Summary of the clustered affected-area groups

\begin{tabular}{|c|c|c|c|c|c|}
\hline \multicolumn{2}{|r|}{$t=1$} & \multicolumn{2}{|r|}{$t=2$} & \multicolumn{2}{|r|}{$t=3$} \\
\hline priority & $\begin{array}{l}\text { The number } \\
\text { of disaster-hit } \\
\text { site }\end{array}$ & priority & $\begin{array}{l}\text { The number of } \\
\text { disaster-hit site }\end{array}$ & priority & $\begin{array}{l}\text { The number of } \\
\text { disaster-hit site }\end{array}$ \\
\hline 1 & $4,5,10$ & 1 & 5、 10 & 1 & 5、 10 \\
\hline 2 & $3,8,9$ & 2 & $3 、 4 、 9$ & 2 & $3 、 4,8,9$ \\
\hline 3 & $1,2,7,12$ & 3 & $1,2,6,7,8$ & 3 & $1 、 2 、 7$ \\
\hline 4 & $6 、 11,13$ & 4 & $11 、 12 、 13$ & 4 & $6,11,12,13$ \\
\hline
\end{tabular}

\subsection{Solution and Algorithm Design of Aircraft Dispatching Model}

The solution is conducted through transforming the dispatching of many distribution centers into that of only one center before optimization. In this method, the strategy of separating many centers and the selection of optimizing final solution are laid emphasis. The solution is determined with boundary distribution method and heuristic algorithm is adopted in the design. Detailed designing is:

(1) To direct arrange the disaster-hit sites: $L$ permutations of natural number from $1 \sim L$ are direct produced. Each permutation composes a solution and corresponds to a delivery plan. Each element (disaster-hit site) is located to the delivery route of each aircraft one by one according to the 
constraint conditions. If one site cannot be located to the route, the solution will be not feasible.

(2)To assess the solution: the route plan of aircraft dispatched can meet the requirement of load capacity and maximum delivery distance in practice, however, this cannot ensure all the disaster-hit areas can be rescued. For one solution, if all sites can be located into the delivery route, its infeasible route number is 0 , which means this solution is feasible; otherwise, it is not feasible with the infeasible route number of 2 . In addition, considering the constraint of grading disaster-hit sites, penalty function method will be adopted to handle the constraint condition if the aircraft cannot be dispatched one by one according to the grading. Specific evaluation of the solution is:

$$
E=F_{g}(t)+M \times P_{w}+N \times P_{g}
$$

(3) Neighborhood reconnaissance of double exchange is adopted according to the features of solutions.

(4) Termination rule: to terminate after some iteration.

$E$ is the evaluation value. The smaller this value is, the higher the quality of solution will be. $F_{g}(t)$ is the value of objective function while $M$ and $N$ are the number of infeasible route and times of delivery skipping the grade respectively; $P_{w}$ and $P_{g}$ are the weights of infeasible route and of penalty of skipping the grade (bigger positive numbers are selected within the range of objective function).

$$
\max F_{g}(t)=\omega_{r} \times\left[\frac{F_{g}^{1}(t)-F_{g_{1}}^{\min }}{F_{g_{1}}^{\max }-F_{g_{1}}^{\min }}\right]-\omega_{c} \times\left[\frac{F_{g}^{2}(t)-F_{g_{2}}^{\min }}{F_{g_{2}}^{\max }-F_{g_{2}}^{\min }}\right]
$$

$F_{g_{1}}^{\max }$ and $F_{g_{1}}^{\min }$ are the expectation maximum and expectation minimum of $F_{g}^{1}(t) ; F_{g_{2}}^{\max }$ and $F_{g_{2}}^{\min }$ are the expectation maximum and expectation minimum of $F_{g}^{2}(t)$. Both the two weight coefficients $\omega_{r}$ and $\omega_{c}$ are 0.5 .

\subsection{Results Analysis}

The quantity of goods demanded in each disaster-hit site in different periods and information about groups of the disaster-hit sites have been known; assume that there are 2 distribution centers, then the position coordinates are known. the attribute value of aircrafts can be called; the two kinds of aircraft has little difference in their loading volumes which are set as $23 \mathrm{~m}^{2}$ and all attribute values of goods (unit volume of various goods are shown in Table 6).

\begin{tabular}{|c|c|c|c|c|}
\hline type & $\begin{array}{l}\text { the max oil } \\
\text { capacity } \\
/(\mathrm{kg})\end{array}$ & $\begin{array}{l}\text { mean of oil } \\
\text { consumption } \\
\text { rate } /(\mathrm{kg} / \mathrm{kw} \cdot \mathrm{h})\end{array}$ & $\begin{array}{c}\text { mean of } \\
\text { speed } \\
/(\mathrm{km} / \mathrm{h})\end{array}$ & $\begin{array}{l}\text { the maximum of } \\
\text { load } \\
\text { capacity } /(\mathrm{cm})\end{array}$ \\
\hline M171 & 2732 & 0.39 & 230 & $460 \times 250 \times 200$ \\
\hline M8 & 2027 & 0.36 & 180 & $420 \times 240 \times 200$ \\
\hline \multicolumn{5}{|c|}{ Tab.6 The unit volume statistics of relief } \\
\hline \multicolumn{2}{|c|}{$\begin{array}{c}\text { material } \\
\text { variety }\end{array}$} & $U_{2}$ & $U_{3}$ & $U_{4}$ \\
\hline $\begin{array}{c}\text { unit } \\
\text { volume } /(\mathrm{cm})\end{array}$ & $m)$ & $30 \times 20 \times 5$ & $50 \times 40 \times 30$ & $120 \times 120 \times 30$ \\
\hline
\end{tabular}

Tab.5 The properties of aircrafts 
Tab.7 The position coordinates of affected points and distribution centers (1:1500000) and corresponding relief demand

\begin{tabular}{|c|c|c|c|c|c|c|c|c|}
\hline $\begin{array}{l}\text { The number of } \\
\text { disaster-hit site }\end{array}$ & 1 & 2 & 3 & 4 & 5 & 6 & 7 & 8 \\
\hline $\mathrm{X}$-axis/(cm) & 2.96 & 6.52 & 7.27 & 14.9 & 7.04 & 6.14 & 14.45 & 1.29 \\
\hline Y-axis/(cm) & 13.36 & 18.82 & 5.26 & 16.45 & 14.25 & 5.03 & 12.08 & 1.42 \\
\hline $\begin{array}{l}\text { material } \\
\text { demands } \\
/(\text { Flights })\end{array}$ & 0.2 & 0.2 & 0.4 & 0.5 & 0.6 & 0.1 & 0.2 & 0.3 \\
\hline $\begin{array}{l}\text { The number of } \\
\text { disaster-hit site }\end{array}$ & 9 & 10 & 11 & 12 & 13 & $\begin{array}{l}\text { The first of } \\
\text { distribution } \\
\text { center }\end{array}$ & \multirow{4}{*}{\multicolumn{2}{|c|}{$\begin{array}{l}\text { The second } \\
\text { of } \\
\text { distribution } \\
\text { center } \\
6.03 \\
11.28 \\
\text { abundance }\end{array}$}} \\
\hline $\mathrm{X}$-axis/(cm) & 3.9 & 15.1 & 2.33 & 10.0 & 11.7 & 11.56 & & \\
\hline Y-axis/(cm) & 9.09 & 17.9 & 15.85 & 19.27 & 16.9 & 13.44 & & \\
\hline $\begin{array}{l}\text { material } \\
\text { demands } \\
\text { /( Flights) }\end{array}$ & 0.3 & 0.5 & 0.1 & 0.2 & 0.1 & abundance & & \\
\hline
\end{tabular}

Considering the influence of routes, $P_{w}$ is set as 500 and $P_{g} 50$ in this paper and the satisfaction rate is above 0.96 . Results of goods distribution and aircraft routes are shown in Table 8 (starting number marks the number of distribution center):

Tab.8 Relief distribution and path selection of aircrafts

\begin{tabular}{|c|c|c|c|c|c|}
\hline The number & \multicolumn{2}{|c|}{$\begin{array}{l}\text { The first of distribution } \\
\text { center }\end{array}$} & \multicolumn{3}{|c|}{$\begin{array}{l}\text { The second of distribution } \\
\text { center }\end{array}$} \\
\hline $\begin{array}{l}\text { The number of } \\
\text { disaster-hit site }\end{array}$ & $2,4,7,10,12$, & & $1,3,5,6,8$ &, 11 & \\
\hline type & M171 & M8 & M171 & M171 & M8 \\
\hline route & $\begin{array}{l}1-2-12-13- \\
7-1\end{array}$ & $1-10-4-1$ & $\begin{array}{l}2-9-8-6- \\
2\end{array}$ & $\begin{array}{l}2-5-11-1 \\
-2\end{array}$ & $2-3-2$ \\
\hline mile: & 338.02 & 174.81 & 351.72 & 236.47 & 184.5 \\
\hline Total of mileage $/ \mathrm{km}$ & 512.83 & & 772.69 & & \\
\hline
\end{tabular}

\section{Conclusions}

Based on forecast of relief goods demand and disaster-hit areas grouping, a planning model of dispatching relief goods is established in this paper and the efficiency of algorithm proposed is verified with simulation data.

(1) The disaster-hit sites are divided into common, urgent, serious and very serious groups, which higher priority of seriously-stricken areas are given, making the rescue and relief more efficient ;

(2) Urgency of goods needed and characteristics of aircrafts are taken into consideration into the objective function, the ability of dispatching aircrafts is put into full play on the precondition of firstness of time.

(3) The expression, generation and saving mode of solutions in the algorithm are simple and practicable, taking up small memory space and the calculation is quite efficient.

However, all the aircraft routes in this paper are straight lines; air routes influenced by severe weather and complex landform are not fully considered, and air conflicts of free aircrafts and its solving plan are not taken into consideration, all of which are the problems needed to be solved in further studies. 


\section{Acknowledgments}

This study was supported by the National Science Foundation of China (no.U1233101, and no.71271113).

\section{References}

[1] Liu Da-xiang, Wang Xiang-sui. The practicable technique way of earthquake prediction -Thoughts on developing China's air emergency rescue ability. Engineering Science, Vol.11 (2009) No.6,p.68-73.

[2] GAO Jian, ZHANG Bing. The status quo of aviation emergency rescue in foreign countries andthe revelations. China Civil Aiviation, Vol.118 (2010) No.1, p.22-24.

[3] Liu Ping. Building an independent aviation rescue system in civil aviation industry.China Civil Aviation, Vol.7 (2008) No.91,p.16-18, .

[4] Barbarosoglu G, Arda Y. A two-stage stochastic programming framework for transportation planning in disaster response.Journal of the Operational Research Society, Vol.55 (2004) No.1,p.43-53,.

[5] Chang M.S, Tseng Y.L, Chen J.W. A scenario planning approach for the flood emergency logistics preparation problem under uncertainty. Transportation Research Part E, Vol. 43, (2007) No.6,p.737-754.

[6] Beraldi P, Bruni M E. A probabilistic model applied to emergency service vehicle location .European Journal of Operation Research, Vol.196 (2009) No.1,p.323-331

[7] Araz C, Selim H J, Ozkarahan I. A fuzzy multi-objective covering-based vehicle location

[8] model for emergency services. Computers and Operations Research, Vol.34,(2007) No.3,p.705-726.

[9] Tzeng G H, Cheng H J, Huang T D.Multi-objective optimal planning for designing relief delivery system. Transportation Research Part E, Vol.43,(2007) No.6,p.673-686.

[10]ZHAN Sha-lei, LIU Nan. Multi-objective stochastic programming model for relief allocation based on disaster scenario information updates.Systems Engineering -Theory \& Practice, Vol.33 (2013) No.1,p.159-166,

[11]Vanda De Angelis, Mariagrazia Mecoli, Chris Nikoi, Giovanni Storchi. Multi-period integrated routing and scheduling of World Food Programme cargo planes in Angola. Computers \&Operations Research, Vol.34,(2007),1601-1615

[12]Bodin.L, Golden.B, Assad.A. Routing andScheduling of Vehicle and Crews:The state of the art.Computers \& Operations Research, Vol.2 (1983) No.10,p.63-211,

[13]Yang Rong-sheng, Pan Wei-jun, Kong Jin-feng.The scheme of establishing and operating civil aviation emergency rescue operation center.China Civil Aviation, Vol.6 (2011) No.126,p.28-31.

[14]Pssquier M, Geiser V, De Riedmattrn M., Carron PN. Helicopter rescue operations involving winching of an emergency physician, Injury, Vol.43,(2012) No.9,p.1377-1380

[15]Zhang Xin, Zhong Jun, Luo Jin-zhou, Li Peng, Zhang Yan-ping. Emergency Support of Air Transportation for Earthquake Relief Work; Its Characteristics and Our Counter-measures. Traffic Engineering and Technology for National Defence. (2008) No.6,p.14-17.

[16]XIA Zheng-hong, PAN Wei-jun, LIN Rui-chun, CHEN Tong. Research on efficiency of aviation emergency rescue under major disasters.Computer Engineering and Design, Vol.3 (2012) No.33,p.1251-1256. 
[17]Liu-lang. Research on Coordination Mechanism between Military and Locals of Aviation Emergency Logistics Response for Unconventional Disruption .Journal of Beijing Institute of Technology(Social Science Edition), Vol.14 (2012) No.22,p.93-99. 